\title{
\begin{tabular}{|l|l|l|l|l|}
\hline M & R & S & Internet Journal of & Nitride Semiconductor Research \\
\hline
\end{tabular}
}

Volume 1, Article 23

\section{Raman Determination of the Phonon Deformation Potentials in a-GaN}

\author{
F. Demangeot, J. Frandon, M. A. Renucci \\ Laboratoire de Physique des Solides de Toulouse, Universite Paul Sabatier \\ Olivier Briot, Bernard Gil, Roger-Louis Aulombard \\ Groupe d'Etude des Semiconducteurs, GES-CNRS
}

This article was received on June 4, 1996 and accepted on October 24, 1996.

\begin{abstract}
Raman spectroscopy is used to study the effect of the built-in biaxial stress on the E2 and A1 (LO) q=0 phonon modes of wurtzite GaN layers deposited by metal organic vapor phase epitaxy on (0001) sapphire substrate. By means of phonon frequency shifts, the biaxial pressure coefficients of the mode frequencies are determined and used to calculate the corresponding deformation potentials. Stress calibration has been performed using reflectance data.
\end{abstract}

\section{Introduction}

Recent breakthroughs in modern epitaxy techniques have enabled the synthesis of GaN epilayers with improved mobility and reduced residual $n$-type conductivity. N-type doping can be reproduceably controlled over several decades using Si donors. The absence of an efficient p-type dopant has long hampered the progress of GaN pn junction based device development. Recently, the successful activation of $\mathrm{Mg}$ acceptors in the GaN lattice by post growth treatments such as LEEBI [1] or in-situ thermal treatment under nitrogen [2] has enabled great progress in GaN based devices.

Optical spectroscopy techniques have long been recognized as efficient methods of investigating semiconductors and their heterostructures. The Raman method is known to be a sensitive technique, in comparison to photoluminescence or reflectance, for determining strains. It can therefore be used to calibrate, mutatis mutandis, the residual strain in ternary alloy layers such as InGaN, which are important for nitride based heterostructures.

Here, we investigate the strain of heteroepitaxial GaN epilayers. These layers, grown on $\left(\begin{array}{llll}0 & 0 & 0 & 1\end{array}\right)$ sapphire substrates using the MOVPE technique, have residual strains present resulting from the lattice and thermal mismatch between the substrate and epilayer. This stress is calibrated using reflectance measurements of the excitonic structure. Next, we determine the deformation potentials for some of the Raman-active phonons in $\alpha-G a N$ epilayers.

\section{Stress calibration}

Reflectance measurements enable the details of the MOVPE GaN/sapphire valence band structure to be probed. In particular, we relate the exciton energies and their splitting to the residual strain in the layers. The complete nature of the residual strain is not clear at the moment. It is weakly related to the sample thickness, and is rather strongly influenced by the layer's growth conditions such as the V/III molar ratio.

Taking into account the spin-orbit and wurtzite crystal field interactions, three excitons (A, B and C) are expected, corresponding to the three electronic transitions, $\Gamma_{7 \mathrm{C}}-\Gamma_{9 \mathrm{~V}}, \Gamma_{7 \mathrm{C}}-\left(\Gamma_{7 \mathrm{~V}}\right)+$ and $\Gamma_{7 \mathrm{C}}-\left(\Gamma_{7 \mathrm{~V}}\right)-$. Careful examination of reflectance spectra reveals an increase of the $A-B$ and $B-C$ energy splittings with increasing energy of the $A$ exciton which is evidence of residual strain fields in the GaN. When the three excitons are plotted as a function of the 
fundamental (A) transition energy, a linear variation with different slopes is obtained. In the case of a cubic approximation, and neglecting spin-orbit interaction, we get expressions for these energies as function of stress. The zero stress values are introduced corresponding to strain free situation. After some elementary manipulations of these expressions and using hydrostatic pressure coefficient, a stress calibration is obtained.

\section{GaN Phonon Spectra}

Wurtzite GaN/sapphire epilayers have symmetry belonging to the C6v4 (P63mc) space group with two formula units per primitive cell. A group theory analysis of $q=0$ lattice vibrations predicts six optical modes which decompose into the following representations of the C6v $(6 \mathrm{mmm})$ point group: $\Gamma_{\mathrm{opt}}=\mathrm{A} 1+\mathrm{E} 1+2 \mathrm{E} 2+2 \mathrm{~B} 1$. The polar modes, $\mathrm{A} 1$ and E1 (both infra-red and Raman active), are polarized along the $z$ optical axis and in the basal (x,y) plane, respectively $(x, y, z$ represent the crystal principal axes). Of the non polar lattice vibrations, the E2 modes are Raman active while the B1 modes are not. Obviously, the anisotropic structure of $\mathrm{GaN}$ and the ionic character of the crystal bonding have a strong influence on the dynamical properties. The anisotropy in short range atomic forces is thus responsible for the A1-E1 splitting while the long-range Coulomb field causes the longitudinal-transverse (LO-TO) splitting of polar modes. This results in direction dependent frequencies and polarization properties when approaching the limiting $q=0$ value along or perpendicular to the optical axis.

The effect of stress on $q=0$ phonons in the wurtzite structure was addressed in piezospectroscopic studies of CdS [3] within the context of a perturbative approach. In the linear approximation, the phenomenological Hamiltonian can be expressed as

$$
\mathrm{H} \varepsilon=V_{\mathrm{ij}} \varepsilon_{\mathrm{ij}}
$$

where the $\varepsilon_{\mathrm{ij}}(\mathrm{i}, \mathrm{j}=\mathrm{x}, \mathrm{y}, \mathrm{z})$ is the strain tensor. Rearranging this contracted tensorial product to form combinations of $V_{\mathrm{ij}}$ transforming according to the $\mathrm{A} 1, \mathrm{E} 1$, and $\mathrm{E} 2$ irreducible representations of the crystal point group leads to the construction of meaningful quantities, i.e. the deformation potentials. The changes in frequency of $q=0$ phonons under stress are obtained in first order perturbation theory in terms of deformation potential constants. Under hydrostatic or biaxial ( $\left.\begin{array}{llll}0 & 0 & 0 & 1\end{array}\right)$ oriented stress, the shifts of the phonon frequencies can be expressed as:

$$
\Delta v \mathrm{~J}=2 \mathrm{a}_{\mathrm{J}} \sigma_{\mathrm{xx}}+\mathrm{b}_{\mathrm{J}} \sigma_{\mathrm{zz}}, \mathrm{J}=\mathrm{A} 1, \mathrm{E} 1 \text {, or E2 }
$$

The $\sigma_{\mathrm{ii}}$ 's are the diagonal components of the stress tensor, with $\sigma_{\mathrm{xx}}=\sigma_{\mathrm{zz}}$ for the hydrostatic pressure and $\sigma_{\mathrm{zz}}=0$ for biaxial $\left(\begin{array}{llll}0 & 0 & 0 & 1\end{array}\right)$ stress. The coefficients $a_{\mathcal{J}}^{\prime}$ and b'J are expressed in terms of the elastic constants and deformation potential constants as:

$$
a^{\prime}=a_{J}\left(S_{11}+S_{12}\right)+b_{J} S_{13}
$$

and

$$
b^{\prime} \mathrm{J}=2 \mathrm{a}_{\mathrm{J}} \mathrm{S}_{13}+\mathrm{b}_{\mathrm{J}} \mathrm{S}_{33}
$$

where $a_{J}$ represents the change in frequency of phonon $J$ per unit relative change of length along $z$, and $b_{J}$ is its counterpart along $x$.

According to a recent investigation of the optical properties of GaN/sapphire [4], exciton energies are extremely sensitive to the biaxial compression in the epilayers. The full nature of the residual strains is not yet elucidated. A thermoelastic contribution, originating from the mismatch of the thermal expansion coefficients between the layer and the substrate, is present, but an additionnal strong dependence on growth conditions (more precisely, the V/III molar ratio) was also established [5].

\section{Vibrational properties in a-GaN under stress}

The $\mathrm{q}=0$ phonon frequencies were measured by Raman spectroscopy (backscattering configuration) in a series of layers grown under different V/III ratios. Stress calibration for each sample was obtained from reflectance measurements as described above. Values of the biaxial stress coefficient $K B_{J}=d v_{j} / d \sigma$ where $\sigma=-\sigma_{x x},(\sigma>0$ for compression) were estimated from the data. A first relation between the $a_{j}$ 's and bj's, obtained from (3) and (4), can thus be established: 


$$
2 a_{J}\left(S_{11}+S_{12}\right)+2 b_{J} S_{13}=-K B_{J}
$$

One more relation is needed for the determination of the $\left\{a_{J}, b_{J}\right\}$ set of deformation potential constants. It is obtained from the Raman measurements carried out by Perlin et al. [6] on bulk GaN crystals under hydrostatic pressure since, according to (3), (4) and (5):

$$
2\left(S_{11}+S_{12}+S_{13}\right) a_{J}+\left(2 S_{13}+S_{33}\right) b_{J}=-K H_{J}
$$

\section{Raman experiment and discussion}

Raman measurements were performed at $77 \mathrm{~K}$ on the series of GaN layers grown at different V/III molar ratios (for further details about growth, see [7]). The layer characteristics are given in Table I, together with the corresponding built-in stress estimated from the $2 \mathrm{~K}$ reflectance studies [5]. The stress at $2 \mathrm{~K}$ can be used confidently at $77 \mathrm{~K}$ also, as demonstrated on Figure 1, which compares the reflectivity spectra recorded on the same sample at $2 \mathrm{~K}$ and $77 \mathrm{~K}$, respectively. Except for an overall red shift of $4 \mathrm{meV}$, and in terms of splittings between $\mathrm{A}, \mathrm{B}$ and $\mathrm{C}$ lines, the $77 \mathrm{~K}$ data is identical to that taken at $2 \mathrm{~K}$. Since the change of the A-C splittings amounts to $1.4 \mathrm{meV} / \mathrm{kbar}$ [5], within the experimental uncertainty in the determination of energy of $C$ line $(<0.5 \mathrm{meV})$ we estimate the error in stress calibration to be at worst 250 bar.

The Raman spectra were excited in backscattering geometry along [ $\begin{array}{llll}0 & 0 & 0 & 1\end{array}$ ], using the $488 \mathrm{~nm}$ radiation of an $\mathrm{Ar}^{+}$ laser. The scattered light was analyzed with a T800 triple spectrometer equipped with a conventional photon counting system. Although the actual scattering geometry allows the observation of the A1 (LO) extraordinary phonon and that of the two E2 modes, the low frequency E2 mode at $144 \mathrm{~cm}^{-1}$ was hardly detected due to far off-resonant conditions with respect to the yellow photoluminescence band. No reliable conclusions can be drawn concerning this mode. Figure 2 shows the non-analyzed Raman spectra in the frequency range of the highest E2 mode. A shift of the E2 frequency is clearly observed with respect to the sapphire line at $578 \mathrm{~cm}^{-1}$, corresponding to mode hardening with decreasing V/III ratio. To a lesser extent, the same trend is noted for the A1 (LO) mode. The phonon frequency as a function of biaxial stress in GaN is deduced from our Raman measurements for the E2 and A1 (LO) modes and is displayed in Figure 3. Using elastic compliance constants obtained from the elastic stiffness coefficients $\mathrm{C}_{11}=296 \mathrm{GPa}, \mathrm{C}_{12}=130 \mathrm{GPa}, \mathrm{C}_{13}=158 \mathrm{GPa}, \mathrm{C}_{33}=267 \mathrm{GPa}$ [7], a least squares fit gives the following values for the slopes: $\mathrm{K}_{\mathrm{E} 2 \mathrm{~B}}=2.9 \pm 0.3 \mathrm{~cm}^{-1} / \mathrm{GPa}$ and $\mathrm{K}_{\mathrm{A} 1(\mathrm{LO}) \mathrm{B}}=0.8 \pm 0.4 \mathrm{~cm}^{-1} / \mathrm{GPa}$. Despite the large imprecision in the $\mathrm{K}_{\mathrm{A} 1 \text { (LO)B }}$ pressure coefficient due to the rather weak Raman efficiency of the mode, its lower value undoubtedly reflects the weak influence of the in-plane biaxial stress to the A1(LO) compared to the E2 mode.

The hydrostatic pressure coefficients of the modes were taken from [6]. Since their heavily doped sample had no observable A1(LO) mode in backscattering geometry along [ $\left.\begin{array}{llll}0 & 0 & 0 & 1\end{array}\right]$, we adopted the pressure coefficient of their $\mathrm{A} 1$ (TO) mode, in fact the phonon-like component of the $\mathrm{A} 1$ (LO) plasmon coupled mode [8]. For $\mathrm{K}_{\mathrm{E} 2 \mathrm{H}}=4.17 \mathrm{~cm}$

$-1 / \mathrm{GPa}$ and $\mathrm{K}_{\mathrm{A} 1(\mathrm{LO}) \mathrm{H}}=4.06 \mathrm{~cm}^{-1} / \mathrm{GPa}$, we obtain the two sets of deformation potential constants, listed in Table II for each mode. As expected, they are two orders of magnitude smaller than those describing strain effects on the electronic band extrema [6]. However, in view of the uncertainty in the $\mathrm{K}_{\mathrm{A} 1 \text { (LO)B }}$ coefficient value, the determination of the deformation potentials of $\mathrm{A} 1$ (LO) mode must be considered only as a tentative one. Anyhow, we are confident in the low value of this coefficient which is indicative of significant anisotropy in the A1(LO) deformation potentials.

\section{Conclusion}

Raman investigations of strained GaN epilayers grown with strain on sapphire substrates have been performed at low temperature. Taking into account the stress calibrations based on reflectance measurements, we determined deformation potentials of two $\mathrm{q}=0$ phonons from shifts in phonon frequencies under the built-in stress. Until now, these deformation potentials have not been obtained for $\mathrm{GaN}$ because classical piezospectroscopic studies under external uniaxial stress require large size crystals not available at this time in GaN. This work should be a helpful basis for further Raman studies of residual strain fields in InGaN- and AIGaN-based heterostructures.

\section{References}

[1] H. Amano, M. Kito, K. Hiramatsu, I. Akasaki, Jpn. J. Appl. Phys. 28, L2112 (1989). 
[2] S. Nakamura, T. Mukai, M. Senoh, N. Iwasa, Jpn. J. Appl. Phys. 31, L139-L142 (1992).

[3] R. J. Briggs, A. K. Ramdas, Phys. Rev. B 13, 5518 (1976).

[4] B Gil, O Briot, RL Aulombard, Phys. Rev. B 52, R17028-17031 (1995).

[5] O. Briot, J. P. Alexis, B. Gil, R. L. Aulombard, Mater. Res. Soc. Symp. Proc. 395, 207-212 (1996).

[6] Piotr Perlin , Claude Jauberthie-Carillon, Jean Paul Itie, Alfonso San Miguel, Izabella Grzegory , Alain Polian , Phys. Rev. B 45, 83-89 (1992).

[7]Science and Technology-Semiconductors: Group IV Elements and III-V Compounds. edited by O. Madelung, (Springer-Verlag, Berlin-Heidelberg, 1991)

[8] P. Perlin , J. Camassel, W. Knap, T. Taliercio, J. C. Chervin , T. Suski, I. Grzegory, S. Porowski , Appl. Phys. Lett. 67, 2524-2526 (1995).

\section{Table I}

Layer characteristics and corresponding built-in stress estimated from $2 \mathrm{~K}$ reflectance experiments.

Sample V/III ratio Growth temperature $\left({ }^{\circ} \mathrm{C}\right)$ Stress $(\mathrm{GPa})$

$\begin{array}{llll}\text { A } & 2000 & 980 & 0.81 \\ \text { B } & 3000 & 1010 & 0.67 \\ \text { C } & 4000 & 980 & 0.48 \\ \text { D } & 5000 & 980 & 0.23 \\ \text { E } & 6000 & 980 & 0.12\end{array}$

\section{Table II}

Phonon deformation potentials of $\mathrm{GaN}$ at liquid nitrogen temperature in $\mathrm{cm}^{-1} /$ unit strain.

Phonon symmetry a b

E2 $-818 \pm 14-797 \pm 60$

A1(LO) $\quad-685 \pm 38-997 \pm 70$ 


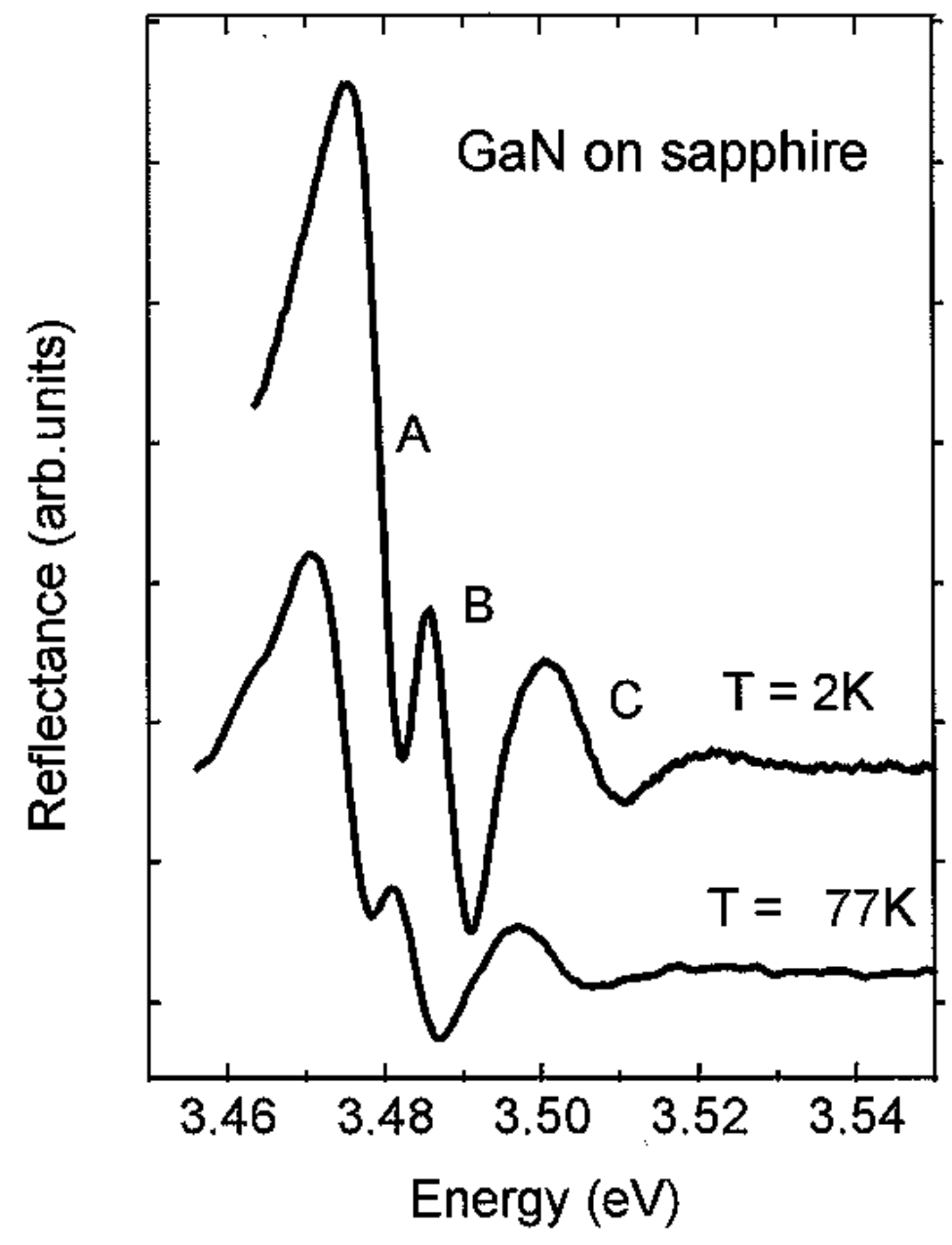

Figure 1. Typical GaN reflectivity spectra taken at $2 \mathrm{~K}$ (full line) and 77K (dashed line). The 77K spectrum is blue-shifted by $4 \mathrm{meV}$ to match the energy of structures A. Except for a weak broadening of the structures with increasing temperature, due to thermal increasing of collisions, the constant splittings we observe between the $A, B$ and $C$ lines is experimental evidence of a constant biaxial strain for temperatures ranging between $2 \mathrm{~K}$ and $77 \mathrm{~K}$. 


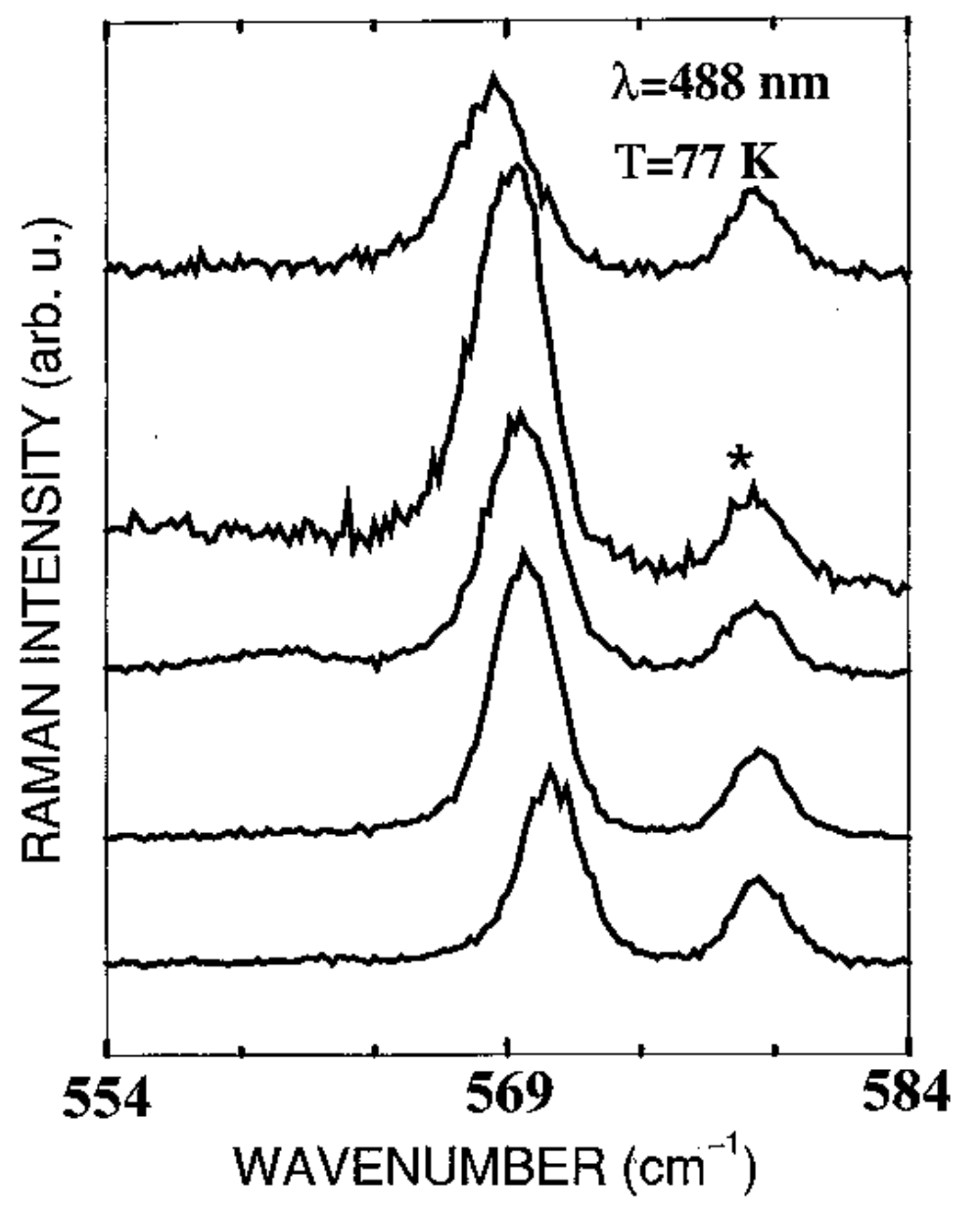

Figure 2. Unanalyzed Raman Spectra recorded at $77 \mathrm{~K}$ for different $\mathrm{GaN}$ layers at $\lambda \mathrm{L}=488 \mathrm{~nm}$ in the E2 frequency range. The asterisk marks one of the sapphire lines at $578 \mathrm{~cm}^{-1}$. 

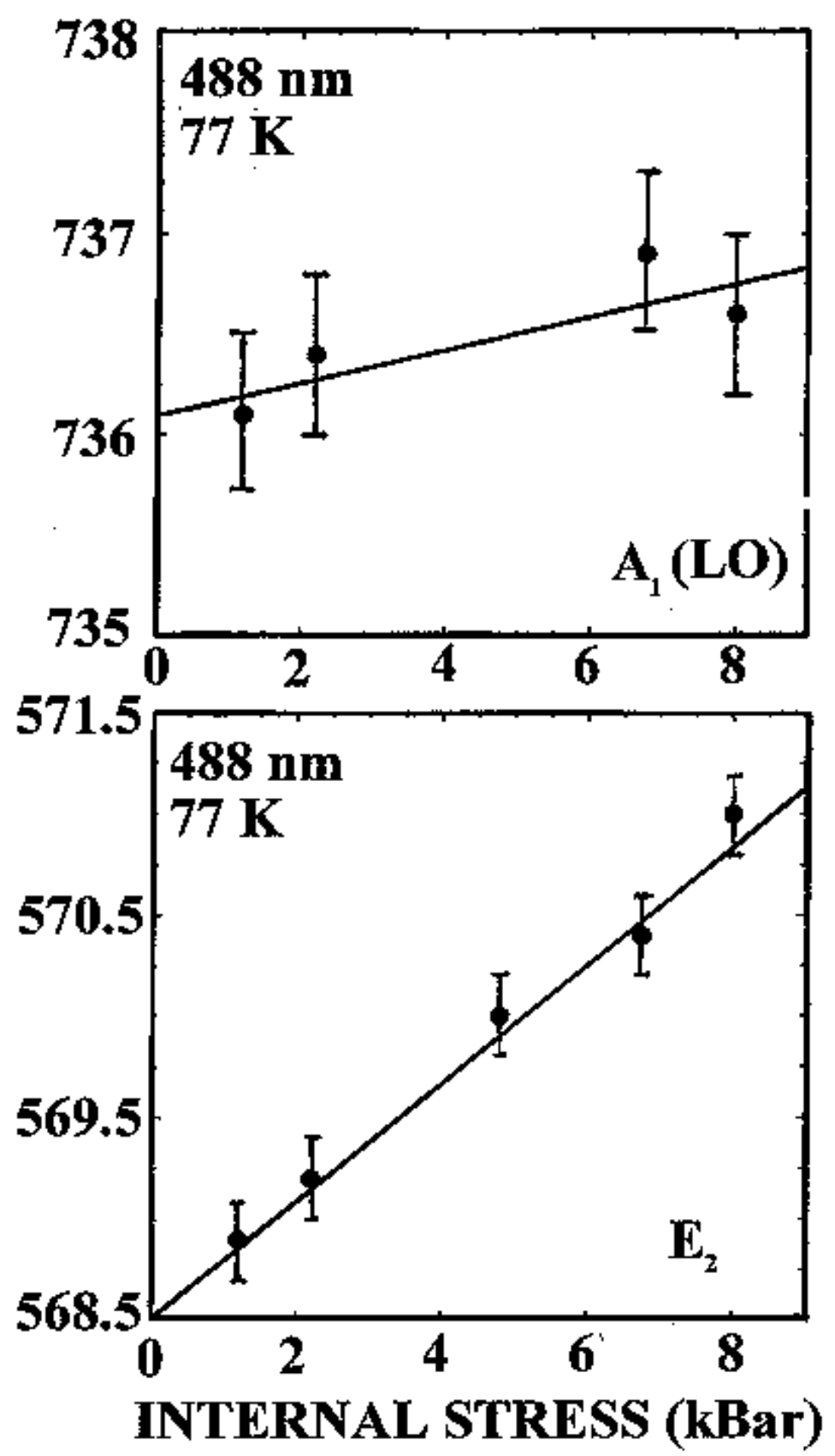

Figure 3. Least-squares fit of the mode wave numbers vs. the internal stress estimated at $77 \mathrm{~K}$ for the E2 and A1 (LO) modes.

(C) 1996-1997 The Materials Research Society

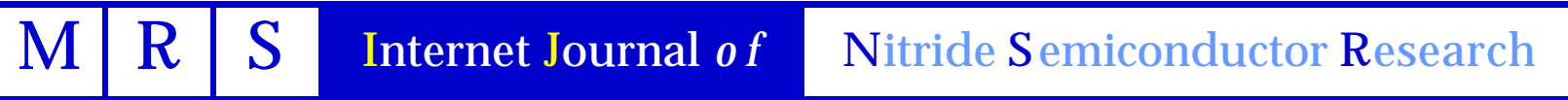

